ORIGINAL PAPER

\title{
Three-dimensional computer-aided reconstruction of FMRFamide immunopos- itive neuron distribution in the ventral ganglion of the barnacle Balanus amphitrite (Cirripedia, Crustacea)
}

\author{
L. Gallus, A. Diaspro' , F. Beltrame 2 , M. Fato² and G. Tagliafierro
}

Dipartimento di Biologia Sperimentale, Ambientale ed Applicata, Università di Genova, Viale Benedetto XV, 5, I16132 Genova, Italy; 'INFM/Dipartimento di Fisica, Università di Genova, Via Dodecaneso 33, I-16146 Genova, Italy and ${ }^{2}$ DIST, Università di Genova, Viale Causa 13, I-16145 Genova, Italy

Accepted: 15/10/00

Key words: three-dimensional reconstruction, crustacea, immunohistochemistry, neuropeptides

\section{SUMMARY}

We have implemented a simple program to solve three of the problems related to 3D reconstruction (3D-Rec) of soft tissues: alignment of sections, distortions, and estimation of the spatial position of elements of interest inside the tissues. As a model, we chose the distribution of FMRFamide-like immunopositive neurons in the ventral ganglion of the barnacle Balanus amphitrite collected during different seasonal periods. Images of immunostained sections were acquired by means of a CCDcamera-equipped microscope and a PC and the reference points were taken inside the sections. The FMRFamide-like immunopositive neurons detected in the barnacle ventral ganglion were grouped into four different classes according to size, shape and staining intensity. More numerous FMRFamide-like immunopositive neurons were detected in the autumn-collected barnacle than in the summer counterpart. The two 3D reconstructions obtained from transverse and longitudinal ventral ganglion sections were efficaciously compared after $90^{\circ}$ rotation of one of them. Comparison of these two 3D-Rec suggests the presence of at least two groups of FMRFamide-like immunopositive neurons that are seasonally-related and probably involved in reproduction.

\section{INTRODUCTION}

Conventional microscopy yields only two-dimensional (2D) images. This fact implies that the threedimensional (3D) organisation of biologically interesting structures, such as organs or tissues, can be lost or misinterpreted (since the single histological sections are too thin in comparison with the volume of the structures investigated). Since individual sections cannot be representative of an overall situation, it is hard to grasp the structural relationship between different tissues and the spatial distribution of cells. Attempts to solve the problem of 3Dreconstruction (3D-Rec) from serial sectioned images have been made by various authors, who have developed specific programs for computeraided 3D-Rec (Gaunt and Gaunt, 1978; Geraud et al., 1988; Runham et al., 1990; Moss, 1992; Cookson, 1994; Liss and Wiberg, 1997; Weninger et al., 
1998). Most of these methods are time-consuming require expensive hardware and are not directly suitable for morphological research associated with immunohistochemistry and other cytochemical techniques. A method has recently been developed to solve 3D-Rec problems by the use of external fiduciary markers, but it is difficult to use with paraffin- embedded specimens (Streicher et al., 1997). In this paper we present a further procedure designed to solve at least three of the problems related to 3D-Rec when physical sectioning is performed, namely: alignment of sections, distortions, and estimation of the position of the section plane in 3D-space. Our specimens were paraffin-embedded, though the procedure also operates correctly with resin-embedded tissues. In the latter case, owing to the characteristics of the resin, there is less shrinkage and distortion of the soft tissue. In this paper it is not necessary to know the actual size of the specimens, but to maintain constant the eventual artifacts for a useful comparison; this is achieved by the use of the same embedding techniques.

As a model, we chose the spatial distribution of FMRFamide-like immunopositive neurons (FLINs) in the ventral ganglion (VG) of the barnacle Balanus amphitrite. Barnacles are highly specialised Crustacea which have a markedly reduced head and abdomen, and a single ventral ganglion instead of five distinctly separate thoracic ganglia as found in other species of this class (Cornwall, 1953; Gwilliam and Cole, 1979; Gwilliam, 1987; Tagliafierro et al., 1992; 1994; Callaway and Stuart, 1999), with a small number of FLINs (Tagliafierro et al., 1989). The tetrapeptide FMRFamide (PheMet-Arg-Phe- $\mathrm{NH}_{2}$ ) was originally isolated from the ganglia of the clam Macrocallista nimbosa by Price and Greenberg (1977) as a molluscan cardio-excitatory neuropeptide, and subsequently immunohistochemically detected in the nervous system of various invertebrates and vertebrates (Kobierski et al., 1987; Thorndyke, 1989; Vallarino et al., 1995).

\section{MATERIALS AND METHODS}

Twenty adult specimens of $B$. amphitrite of the same size were collected in the Port of Genoa (Italy) during two different seasonal periods. Barnacles were killed by a lethal concentration of MS222 (Sandoz, CH), diluted 1:1000 in sea water.
The bodies of the barnacles were removed from the shell, fixed in Bouin or 4\% paraformaldehyde and embedded in Paraplast (Sherwood Medical, Ireland). Both longitudinal and frontal consecutive sections ( $5 \mu \mathrm{m}$ thick) of the whole barnacle body were cut and mounted on polylysine-coated slides. The sections were immunostained using the indirect immunoperoxidase method (Sternberger, 1979): dewaxed sections were rinsed in 0.2 $\mathrm{M}$ phosphate-buffered saline, $\mathrm{pH} 7.4$ (PBS), pretreated with $2 \% \mathrm{H}_{2} \mathrm{O}_{2}$ in $\mathrm{PBS}$ to eliminate endogenous peroxidase activity, and incubated overnight in a moist chamber, at room temperature, with a polyclonal antiserum raised in rabbit against synthetic-FMRFamide (Affinity, UK), diluted 1:400 in PBS. After rinsing in PBS, the sections were incubated for 1 hour at room temperature with a goat anti-rabbit antiserum conjugated with peroxidase (Dako, DK) diluted 1:100 in PBS. The peroxidase was stained with a fresh solution of 3-3diaminobenzidine-4HCl (DAB) $(30 \mathrm{mg} / 100 \mathrm{ml})$ and $\mathrm{H}_{2} \mathrm{O}_{2}(0.01 \%)$.

To increase the specificity of the method, both positive and negative controls were carried out. Pre-absorption of the antiserum with its corresponding antigen always gave negative results.

\section{Image processing and visualisation}

In order to obtain 3D-Rec of the spatial distribution of immunopositive microscopic biological structures inside tissues or organs, it was necessary to use an integrated imaging system for acquiring, analysing, treating and visualising images of a complete set of serial immunostained sections. This 3D-Rec was performed in three steps:

1) image acquisition and digitalisation;

2) digital image processing;

3) 3D-visualisation by a system of virtual-reality hardware and software.

1) Image acquisition and digitalisation. Complete sets of images obtained from serial sections of a particular biological structure, and a digital image acquisition system, should be used. In this study we used a 486 PC, a Sony DXC-107 P video-camera, and an Olympus optical microscope, model BH2-RFCA (objective lens: 20x, $0.40 \mathrm{NA}$; ocular lens NFK 2.5X LD). The acquisition and quality enhancement of the images were carried out by means of Global Lab Image soft- 
ware, version 3.1 (@1984 Data Translation, Inc. and Acuity Imaging), running under Windows 3.1 and an interface schedule model DT2867 (Data Translation, Inc. USA). All images were originally 8-bit digitised and subsequently recovered into Tagged Image File (TIFF) and Raw. Grey-tone image acquisition was performed using a CCD camera with auto-balance of white-tones, after contrast and integration calibration (Russ, 1995).

2) Digital image processing. The program used for image segmentation and image overlapping was implemented under Matlab (Mathworks Inc). This program runs under UNIX and Windows operating system platform. A PC with a processor of at least 486-DX4 $100 \mathrm{Mhz}$ and 128 Mbytes of RAM is required. Before segmentation, a low-pass medium filter was utilised as critical decision of a particular threshold level. The segmented elements of interest could be the contour-line of the structure studied and the localisation of various objects inside. The contour-line was indicated by thin stroke (a few pixels wide) and the position of the object located inside was indicated by one spot of variable size. In this study both strokes and spots were $100 \%$ definite, with maximum density and without any transparency, fading or spacing. Preferably, they should be marked in a colour that is almost absent from the image. After segmentation, the images must be aligned, as they rarely present the same spatial orientation. This step, called roto-translation, is critical and can be carried out by implementing a second algorithm, which makes it possible to obtain a pile of overlapped images from a set, or few stacks, of consecutive sections. Alignment was effected by using three fiduciary markers, which must be correctly superimposed. These markers usually correspond to extrinsic fiduciaries, like thin threads, inserted into the specimen during inclusion; one of these markers must be used as a hinge while the other two function as a guide and a control. In our specimens, the use of extrinsic fiduciaries would cause relevant damage to the ganglion because of its small size; thus it was necessary to choose internal markers. We used two giant striated muscle fibres running for a sufficiently long tract, and one cuticle pit near the ventral ganglion. These markers were evidenced in the same way as those used to identify the location of the internal objects of interest.

The roto-translation process consisted of three steps.
2.1) Fiduciary identification.

2.2) Roto-translation

2.3) Cropping.

Fiduciary acquisition was directly performed by selecting the fiduciary markers by means of the mouse. The first image acted as a model for the rotation. The file was selected and activated; the first fiduciary marker (the hinge) was selected and then the second one (the guide which imposes the angle of rotation). The same was done for the second image. On selecting the command "rotate" of our program, the second image will be rotated and saved in a new file. By means of the option "compare images", the first image and the second rotated image can be overlapped and visualised to verify the result. While the program rotates the second image for the alignment, it automatically effects the cropping. Cropping is the cutting of those portions of rotated images that cannot be included inside the first image. The images so obtained were transformed into binary files and read as a whole, or stack, by different programs concerning $3 \mathrm{D}$ visualisation.

The result will be the representation of the internal object distribution and the contour-line of the ganglion, reconstructed in a virtual volume.

4) $3 \mathrm{D}$ visualisation. We performed $3 \mathrm{D}$ visualisation by means of the "ray tracing" technique (Diaspro et al., 1990; Diaspro et al. 1996) using the "image application platform" (IAP; ISG Technologies, Toronto, Canada), a software for displaying images interactively. The graphic interface runs under UNIX X-11 OSF/MOTIF, resident on the HP workstation 9000/735. The operator can interact with each image to change the point of view. The processed images may be displayed in stereo vision using liquid crystal glasses (Crystal Eyes, Stereographics Inc., San. Raphael, CA, USA) or with a head-tracker, directly connected with the head of the observer (Beltrame et al., 1995; Diaspro et al., 1996). We prepared two stacks of each set of consecutive sections: one stack displayed the position of the internal objects together with the contour-line, and the other stack displayed the position of the internal objects only, without the contour-line. Alternatively, it is also possible to display the contour-line as a transparent envelope together with the internal object distribution. 


\section{RESULTS}

Using FMRFamide antiserum, a few immunopositive neuron cell bodies and numerous immunopositive nerve terminals were detected in both longitudinal and transversal sections of B.amphitrite ventral ganglion. Most of the immunopositive neurons were located in the cortical portion and only a few in the central neuropil (Figs. 1-2). They were different in size and immunostaining. They were subdivided into four groups, symmetrically localised on either side (Figs. 3-5):

a) faintly immunostained neuron cell bodies of large size $(>30 \mu)$;

b) strongly immunostained neuron cell bodies of medium size and triangular shape $(15-30 \mu)$;

c) neuron cell bodies of small size $(<15 \mu)$;

d) one strongly immunostained neuron cell body of medium size $(15-30 \mu)$ and one small $(10 \mu)$ round neuron cell body;

The serial immunostained sections of the ventral ganglion were then processed for 3D-Rec as indicated in Materials and methods. To reconstruct the spatial distribution of FLINs, we used two complete sets of images obtained from serial immunostained sections of two $B$. amphitrite ventral ganglia, oriented perpendicularly to each other. Instead of physical markers, it was necessary to choose internal markers near the ventral ganglion, such as one cuticle pit and two of the giant striated muscle fibres, which are near the ventral ganglion and characteristic of barnacles, as shown in Fig. 6. The elements of interest to highlight by segmentation were the neural sheet, representing the contour-line, and the FLIN cell bodies, as the internal objects. The contour-line of the ventral ganglion was indicated by a 4-pixel-wide stroke, and the position of each FLIN cell body section by a 8-pixel-diameter spot. Both strokes and spots were red at saturation, $100 \%$ definite, with maximum density and without any transparency, fading or spacing. We used the colour red, corresponding to the grey level 76, because this value was almost absent from our images. We have already explained that fiduciary markers must be used as a hinge, guide and control during alignment (Figs. 7 and 8). The digital images were roto-translated and overlapped as indicated above (Figs. 9 and 10).

The result will be the external view of the ventral ganglion (Fig. 11), the representation of the FLIN cell body distribution inside the ventral ganglion contour-line, reconstructed in a virtual volume (Figs. 12 and 13), and finally the distribution of the FLIN cell bodies, without the external sheet (Figs. 14 -16). The four different groups of neurons identified during microscopic observation were indicated as 8-pixel-diameter spots stained in 4 colours at saturation: blue, green, red, and yellow, respectively. Each spot corresponded to one body neuron section, and the neuron cell bodies were represented by one to three slightly superimposing coloured spots depending of their size. In the autumn-collected barnacles, 3D-Rec (Fig 14) showed 50 immunopositive neurons organised in clusters along the antero-posterior and the dorsoventral axis of the ventral ganglion. The group a (blue) was arranged in two clusters $(\mathbf{a 1}, \mathbf{a 2})$ located in the ventral cortex: one in the median region and the other anteriorly. The group $\mathbf{b}$ (green) was arranged in one cluster located in the posterior region of the dorsal cortex. The group c (red) was the most numerous: the neurons were arranged in 5 clusters, four in the cortex, and one in the central neuropil area. Two clusters (c1, c2) were found in the posterior region, the third (c3) in the mid-dorsal region, the fourth (c4) in the anteroventral region, and the fifth $(\mathbf{c 5})$ in the antero-dorsal region. The four neurons of group $\mathbf{d}$ (yellow) were located in the dorsal cortex, anterior to those of group b (Fig. 14). In the ventral ganglion of summer-collected barnacles, all groups were still present, but they were fewer in number. The 3DRec of the serial immunostained transverse sections of one summer-collected barnacle showed the presence of only 20 FLINs and showed the ventral ganglion in a different perspective, since the orientation for the reconstruction was perpendicular to the previous one. The two 3D-Rec thus obtained were perpendicular to each other, since they came from longitudinal and transverse sections of the ventral ganglion, but they could be compared after 90-degree rotation of one of them by means of the 3D-Display program.

Comparison of the autumn 3D-Rec (Fig. 15) with the summer 3D-Rec (Fig. 16) revealed that in the latter period of the year the immunopositive neurons of groups $\mathbf{a}$ and $\mathbf{c}$ were diminished in number, while a new cluster of immunopositive neurons of group b (indicated as b2) was present in the ventral portion. 

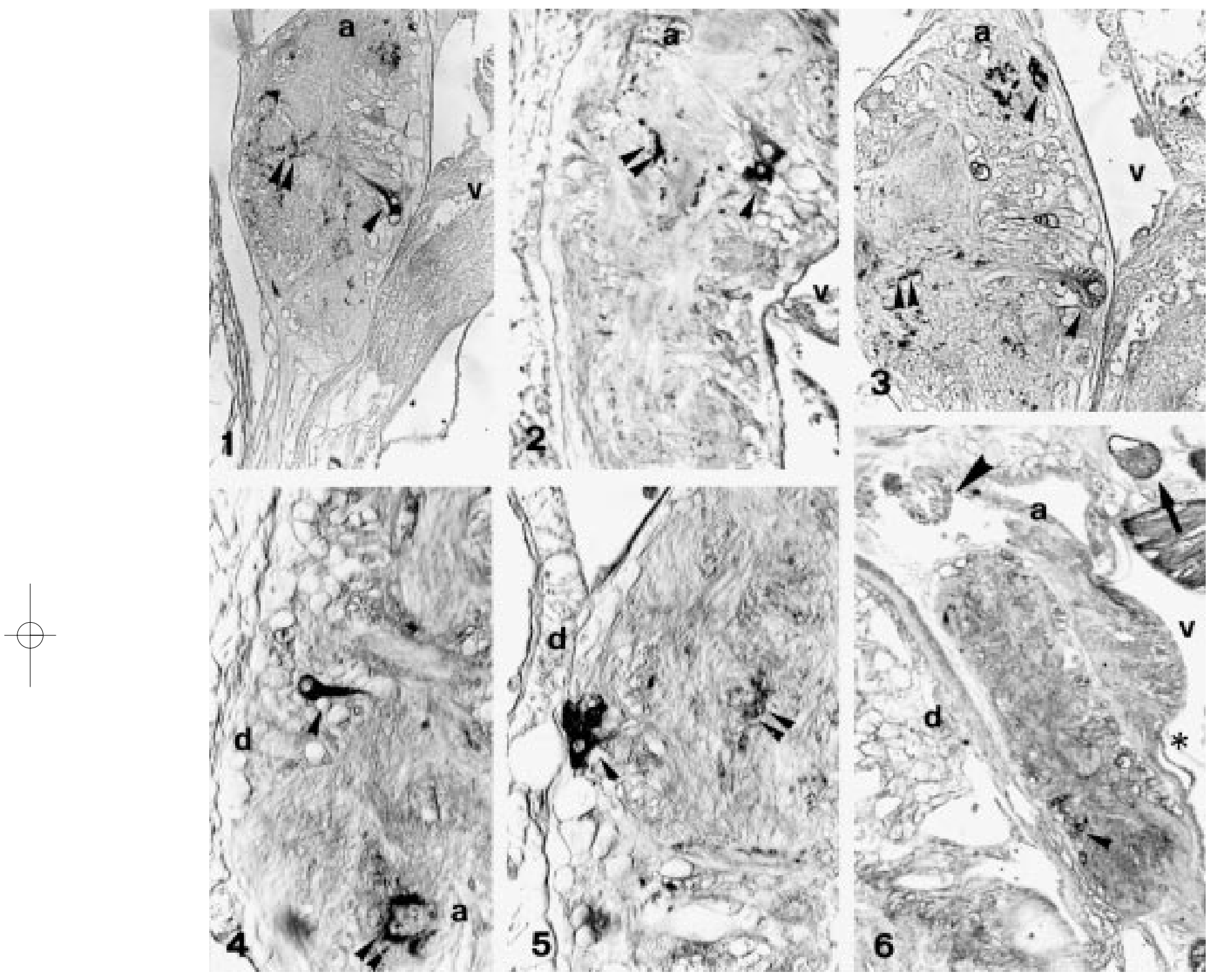

Figs. 1-6 - B. amphitrite, ventral ganglion. FMRF-amide immunopositive neuron cell bodies (small head arrow) and nerve fibres (double small head arrows). v: ventral side, d: dorsal side, a: anterior side. Immunoperoxidase-DAB. (1) Longitudinal section showing one immunopositive neuron of group $\mathbf{b}$ in the cortical ventral region. 150x. (2) Longitudinal section showing two immunopositive neurons of group b near the central neuropil. 250x. (3) Longitudinal section with one immunopositive neuron of group a and two immunopositive neurons of group c. 200x. (4) Longitudinal section with one immunopositive neuron of group b located in the dorsal side. 250x. (5) Transverse section showing three of the four immunopositive neuron cell bodies of group d in the cortical dorsal region. 250x. (6) Autumn-collected barnacle, longitudinal section. The arrowhead represents the muscular fibres used as control marker, the arrow represents the muscular fibre used as guide for rotation, and the asterisk represents the pit of the cuticle used as hinge marker. 100x. 


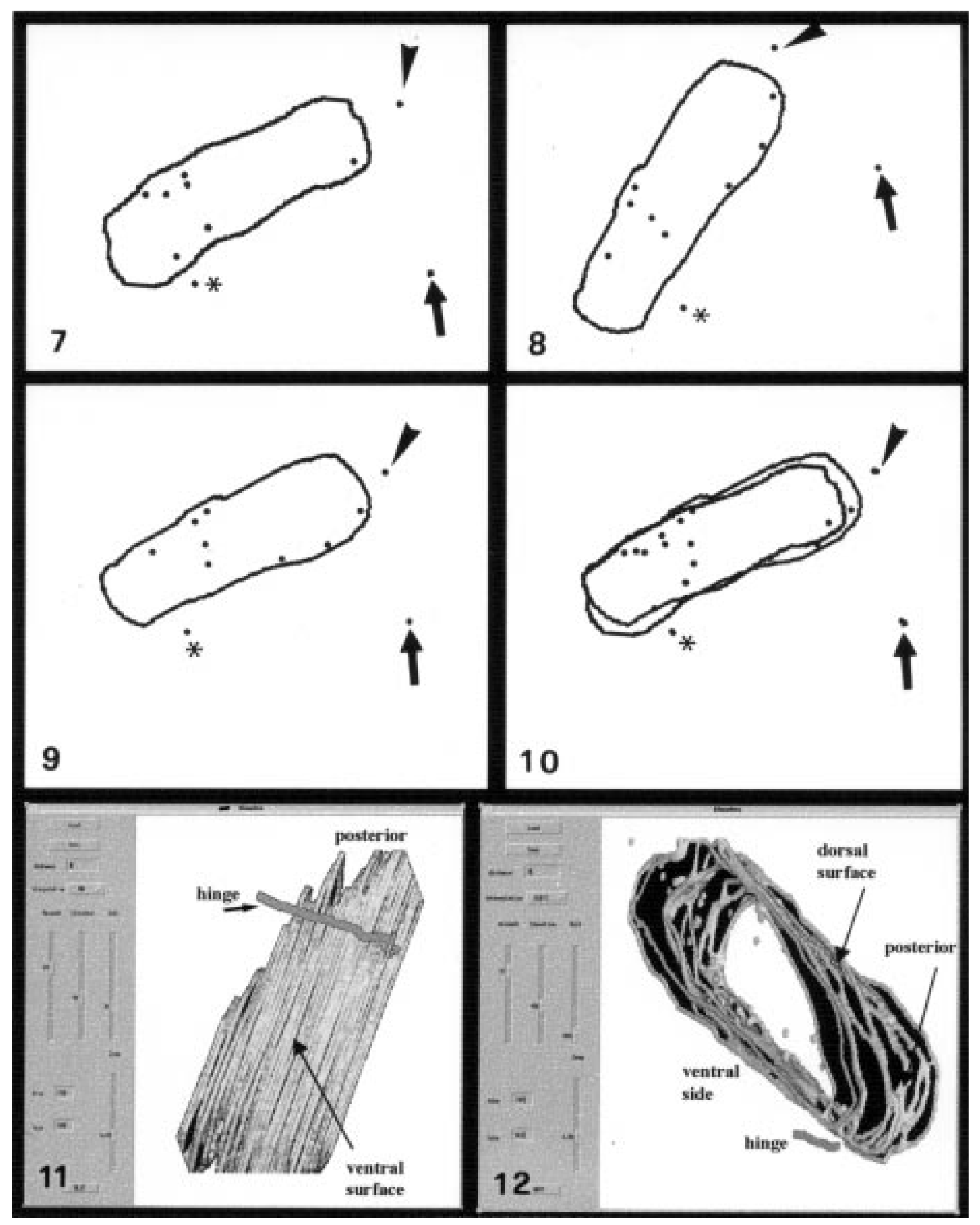




\section{DISCUSSION}

3D-Rec based on serial histological sections is at present the most useful method of obtaining information on the spatial distribution or organisation of one or more particular cell types inside various kinds of biological organs. In the present study, we used an image processing system in order to design a method which requires a minimum of knowledge of computer science. This approach to 3D-Rec from serial sections offers some advantages; one of the most important being that alignment can be carried out objectively and in a semiautomatic way. We aligned the serial sections using virtual fiduciary markers taken inside and near the biological structure studied, instead of the extra-specimen markers used by other authors (Runham et al., 1990; Streicher et al., 1997).

Semi-automatic alignment works better than previous solutions, such as that using physical models or the superimposition of transparent drawings (Geraud, 1988; Weninger et al., 1998); it does not require any particular graphic or artistic aptitude on the part of the operator, who can operate in a standardised and reproducible way. The availability of a well-defined working protocol enables many 3D-Rec of biological objects to be elaborated in a relatively short time, as a large number of images can be easily filed on a PC. External markers have various advantages and are certainly convenient and of great objectivity (Streicher et al., 1997), but they cannot be used if the object of the reconstruction is paraffin-embedded and of small size, such as the barnacle ventral ganglion $(<0.5 \mathrm{~mm})$. The external markers (thin threads or drill holes), even if very close to the barnacle body, are still too far from the ventral ganglion and fall outside the optical field when a 20x objective lens is used. The transverse section of two giant striated muscle fibres (peculiar to the barnacle), which show no variation in size and shape, and run perpendicularly to the ventral ganglion for a convenient distance, can substitute for the external mark- ers very well. The use of internal anatomical landmarks was already successfully performed by Gaunt and Gaunt (1978).

The method illustrated here enables us to reconstruct not only the contour of the object, in this case the neural sheet of the barnacle ventral ganglion, but also the distribution of various structures present inside this contour, i.e. the different groups of FLINs. The random distribution seen in the 2D histological sections is transformed by 3DRec into a well-defined cluster organisation along the ventral ganglion axes. This 3D FLIN distribution is in accord with the fact that the ventral ganglion of the barnacle is derived from the fusion of thoracic ganglia. The small neurons of group $\mathbf{c}$ organised into 5 clusters may correspond to those found anteriorly in every thoracic ganglion of more evoluted Crustacea (Kobiersky et al., 1987).

Comparison of the 3D distribution of FLINs in barnacles collected in different seasonal periods allowed us to suggest that at least three of the FLIN groups showed variation in number and immunopositivity according to the period of the year considered. This variation could be related to reproduction, and in particular to oogenesis. A more accurate quantification and statistical analysis concerning the presence of FLINs in different periods of the year are necessary to confirm this hypothesis, as well as the presence of FMRFamide immunoreactive nerve fibres in the reproductive organs in some periods of the year.

The 3D visualisation method allows the operator to display the 3D-reconstructed structures from different perspectives, and to compare stacks of sections obtained from different sectioning planes (Diaspro et al., 1996). To underline the suitability of this 3D-Rec program, we compared the 3D-Rec obtained from longitudinal ventral ganglion sections with that derived from transverse ventral ganglion sections; although they are orthogonal to each other, they can be compared after rotation of one of them. This possibility constitutes another advantage of the computer aided 3D-Rec, and for

Figs. 7-12 - Computational representation of image processing and visualisation. The first (7) and the second segmented images (8); the contour-line of the ventral ganglion is drawn at the computer by a thin stroke, the three fiduciary markers (the hinge with an asterisk, the guide with one arrow and the control with an arrowhead) and the immunopositive neurons by spots. (9) The second image after roto-translation. (10) Alignment and overlapping of the two images. (11) 3D visualization of the external neural sheet of ventral ganglion, view from the ventral side. (12) 3D representation of the central portion of the ventral ganglion, displaying its neural sheet and the position of the immunopositive neurons, view from the latero-ventral side. 
Imp. Gallus 11-02-2002 16:46 Pagina 102
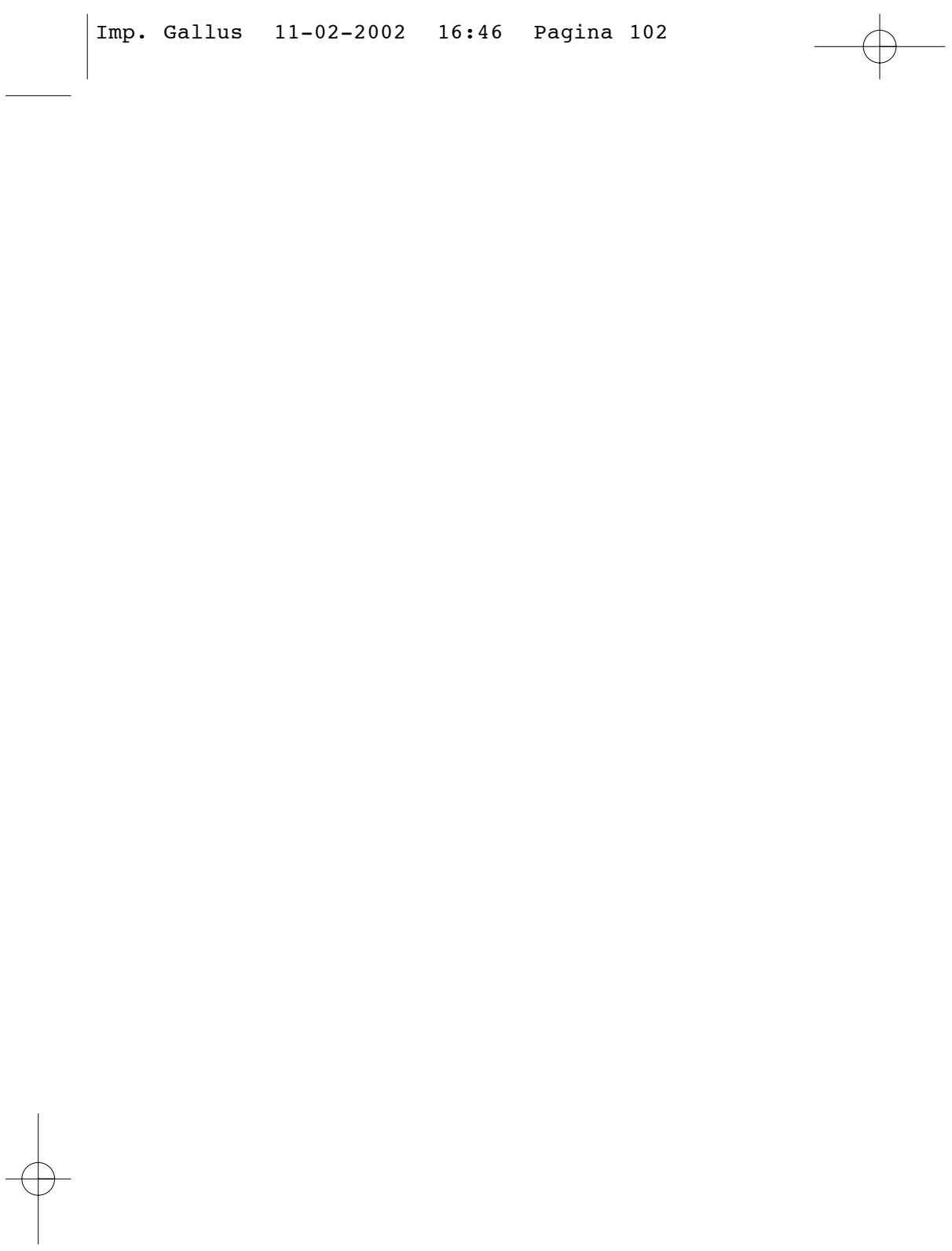
this reason it can be used for specimens embedded without any previously established orientation.

The method we used for the 3D representation is now available for future work related to immunohistochemical, or other molecular biology, and ultrastructural techniques. It is a semi-automatic, time-saving, reproducible tool to gather information of morphological and functional interest regarding the 3D organisation of various kinds of structures inside compact organs.

\section{ACKNOWLEGMENTS}

The research was supported by grants from the University of Genova, Italy.

\section{REFERENCES}

Beltrame F., Diaspro A., Fato M., Martin I., and Ramoino P.: Use of stereo vision and 24-bit false imagery to enhance visualisation of multimodal confocal images. In: Wilson T., Cogswell C.J., editors. Three-dimensional microscopy: image acquisition and visualisation. vol. 2412 Bellingham, USA: SPIE press, pp. 222-229, 1995.

Callaway J.C., and Stuart A.E.: The distribution of histamine and serotonin in the barnacle's nervous system. Microsc. Res. Technique 44, 94-104, 1999.

Cookson J.: Three-dimensional reconstruction in microscopy. Proc. R. Microsc. Soc. 29, 3-10, 1994.

Cornwall I.E.: The central nervous system of barnacles (Cirripedia). J. Fish Res. Board Can. 10, 76-84, 1953.

Diaspro A., Sartore M., and Nicolini C.: 3D representation of biostructures imaged with an optical microscope. Part A, B. Image and Vision Computing 8, 130-141, 1990.

Diaspro A., Beltrame F., Fato M., and Ramoino P.: Characterizing biostructures and cellular events in 2D/3D using widefield and confocal optical sectioning microscopy. IEEE Eng. Med. Biol. 15, 92-100, 1996.

Gaunt P.A., and Gaunt P.N.: Three-dimensional reconstruction in biology. Pitman Medical London, 1978.

Geraud G., Soyer A., and Epelboin Y.: Three-dimensional computer reconstruction from serial sections of cell nuclei. Bio. Cell 62, 111-117, 1988.

Gwilliam G.F., and Cole E.S.: The morphology of the central nervous system of the barnacle, Semibalanus cariosus Pallas. J. Morph. 159, 297-310, 1979.

Gwilliam G.F.: Neurobiology of barnacles. In: Southward AJ, editor. Barnacle biology. Rotterdam: Balkema. pp. 191-211, 1987.

Kobierski L.A., Beltz B.S., Trimmer B.A., and Kravitz, E.A.: FMRFamide-like peptides of Homarus americanus: distribution, immunocytochemical mapping, and ultrastructural localization in terminal varicosities. J. Comp. Neurol. 266, 115, 1987.

Liss A.G., and Wiberg M.: Loss of nerve endings in the spinal dorsal horn after a peripheral nerve injury. An anatomical study in Macaca fascicularis monkeys. Eur. J. Neurosci. 9, 2187-2192, 1997.

Moss VA.: Fundamentals of 3-D reconstruction of serial sections on a microcomputer. In: Kriete A., editor. Visualisation in Biomedical Microscopies -3D imaging and computer applications. Weinheim: VCH. pp. 19-43, 1992.

Price D.A., and Greenberg M.J.: Structure of a molluscan cardioexcitatory neuropeptide. Science 197, 670-671, 1977.

Runham N.W., Davies D.A., and Roberts D.: Computer-aided three dimensional reconstructions from serial section. Microscopy and Analysis (European edition) 14, 15-18, 1990.

Russ J.C.: The image processing handbook, $2^{\text {nd }}$ edn. Boca Raton, USA: CRC Press, 1995.

Sternberger L.A.: Immunocytochemistry, $2^{\text {nd }}$ edn. New York: John Wiley and Sons. 354 p., 1979.

Streicher J., Weninger W.J., and Muller G.B.: External marker-based automatic congruencing: a new method of 3D reconstruction from serial sections. Anat. Rec. 248, 583-602, 1997.

Tagliafierro G., Rossi G.G., and Crosa C.: Neural FMRFamide-like peptide in Balanus amphitrite. Bas. Appl. Histoch. 33 (suppl.), 98, 1989.

Tagliafierro G., Canepa M., Faraldi G., and Zanin T.: Interneural and neuromuscular synapses in the barnacle Balanus amphitrite (Cirripedia, Crustacea). In: Megias-Megias L., Rodriguez-Garcia M.I., Rios A., Arias J.M. editors. Electron microscopy 92, vol. 3. Granada: Universidad de Granada, pp. 715-716, 1992

Figs. 13-16 - 3D representation of the ventral ganglion. (13) 3D representation of the same portion of the ventral ganglion as in Fig. 12, view from the lateral side. The arrowhead indicates the cluster c3. (14) Representation of the distribution of immunopositive neurons in the whole ventral ganglion of one autumn-collected $B$. amphitrite. Each neuron, depending of its size, is represented by one to three superimposing coloured spots; each spot corresponds to one body neuron section. The different clusters are indicated by different groups of coloured spots. The external neural sheet is not shown. Comparison of the distribution of the immunopositive-neurons in the ventral ganglion of two different $B$. amphitrite collected respectively in autumn (15) and summer (16) The arrow puts in evidence the new cluster of immunopositive neurons in the summer-collected barnacle. 
Tagliafierro G., Canepa M., Faraldi G., Toso F.: Enteric neuromuscular junctions in the barnacle Balanus amphitrite (Cirripedia, Crustacea). In: Jouffrey B., Colliex C. editors. Electron microscopy 94, vol. 3. Les Ulis: Les Editions de Phisiques, pp. 667-668, 1994.

Thorndyke C.M.: Peptides in invertebrates. In: Holmgren S., editor. The comparative physiology of regulatory peptides. London: Chapman and Hall, pp. 203-228, 1989.
Vallarino M., Feuilloley M., Thoumas J.L., Demorgny R., Masini A.M., and Vaudry H.: Distribution of FMRFamidelike immunoreactivity on the brain of the lungfish Protopterus annectens. Peptides 16, 1187-1196, 1995.

Weninger W.J., Meng S., Streicher J., and Muller G.B.: A new episcopic method for rapid 3-D reconstruction: applications in anatomy and embryology. Anat. Embryol. 197, 341-348, 1998. 